\title{
Outcome of routine microbiological screening for lower genital tract infections in symptomatic non-pregnant females complaining infertility
}

\author{
Gupta Sapna ${ }^{1}$, Goyal Ankur ${ }^{2}$, Singh Saroj ${ }^{3}$, Agrawal BM ${ }^{4}$ \\ ${ }^{1,2,4}$ Department of Microbiology, SN Medical College, Agra, India \\ ${ }^{3}$ Department of Obs \& Gynae, SN Medical College, Agra, India
}

\begin{abstract}
Worldwide, the risk for infectious vaginitis in young women generally starts as sexual activities begin. These infections may lead to long-term sequels including pelvic inflammatory disease, infertility, and cervical cancer. So the study aimed to establish the different prevalence of lower genital tract infections in symptomatic non-pregnant females complaining infertility attending gynecology clinic. We analyzed total 920 women for the presence of Gardnerella vaginalis, Trichomonas vaginalis, Candida species, Streptococcus agalactiae, Enterobacteriaceae and Enterococci. Their association with presence of lactobacilli and polymorphs were also seen. In our study population, high percentage of isolation of Enterobacteriaceae, Streptococcus agalactiae, and Enterococci with high percentage of antimicrobial resistance against routinely used antibiotics, suggest the importance of vaginal discharge culture and sensitivity in routine practice. We observed highest prevalence of bacterial vaginosis in our study population. Mono-microbial infection was more common than multi-microbial infection. Moreover absence of lactobacilli and presence of polymorphs came out to be important indicators for presence of these infections and to consider the health status of female urogenital tract. Our findings suggest that symptomatic female should be screened for these infections as the consequences may be up to infertility.
\end{abstract}

Key words: Vaginitis; Infertility; Lower genital tract infection; Symptomatic females

\section{Introduction}

Infectious vaginitis is a common problem encountered in clinical medicine which is usually transmitted sexually and is a frequent reason that women visit an obstetrician or gynecologist. The risk for sexually transmitted infections (STIs) in majority of young women is initiated during adolescence as sexual activity starts, most of time the course of the disease is benign, but some infections may lead to long-term sequel, including pelvic inflammatory disease, infertility, and cervical cancer. The pathogenesis in vagina is usually triggered by the imbalance of vaginal micro-environment with superseding of pathogenic micro flora over normal vaginal flora, usually lactobacilli. ${ }^{[1]}$ Physiological or non-physiological factors may affect the vaginal flora. This change in micro flora of female genital tract by pathogenic organisms may ascend from vagina to upper genital tract and cause endometritis, salpingitis and pelvic inflammatory disease (PID), ectopic pregnancy or tubal factor infertility. PID is one of the important public health problem that occur among non-pregnant women of reproductive age with common lower genital tract infections, especially Chlamydia trachomatis, Neisseria gonorrhoeae, and bacterial vaginosis (BV) which may cause long-term sequelae including tubal factor infertility, ectopic pregnancy, chronic pelvic pain, and recurrent PID. ${ }^{[2]}$ Many studies have demonstrated that $\mathrm{BV}$ is associated with the development of pelvic inflammatory disease ${ }^{[3]}$ It is a gynecologic condition of unknown etiology and characterized by decrease in vaginal Lactobacillus sp. accompanied by polymicrobial anaerobic overgrowth. Amsel described BV as a condition with homogeneous grayish-white vaginal discharge, bacterial overgrowth, elevated $\mathrm{pH}$ above 4.5 , and an amine odor released upon addition of $10 \% \mathrm{KOH}$ to the vaginal discharge fluid. ${ }^{[4]}$ Nugent et al. then quantitated the number and types of bacterial overgrowth observed in Gram stained vaginal fluid and developed a Nugent Gram stain score ${ }^{[5]}$ Recently Trichomonas vaginalis infection also has been recognized as associated with preterm birth, cervical neoplasia, posthysterectomy infection, atypical pelvic inflammatory disease and infertility. ${ }^{[6]}$ Trichomonas vaginalis is a flagellate protozoan transmitted sexually and often present as an unpleasant, irritating and potentially dangerous disease leading to important medical, social, and economical implications. Mainly a disease of reproductive years, it persists for long periods in females and characterized by yellowish-green frothy discharge, pruritus, dysuria, dyspareunia, and the "strawberry" cervix. The prevalence of this infection is very high worldwide which may even be underestimated as the infection is mostly asymptomatic in females. Vulvo-vaginal candidiasis is also an important cause of non-transmissible genital infections, but has been viewed not little more than a nuisance. ${ }^{[7]}$ Moreover, some studies have associated aerobic bacterial infections like Enterobacteriaceae, Enterococci and Streptococcus agalactiae in causing vaginal infections that may predispose the spread of infectious agents 
towards the upper female genital tract leading to a predisposition for the development of tubal patency and motility disorders, ovarian function disorders and endometritis. ${ }^{[1]}$ So the aim of this study was to verify our local epidemiology and to establish the different prevalence of various infections in women of reproductive age group complaining infertility.

\section{Methods}

Patients were recruited at a gynecologic outpatient clinic based in a tertiary care centre located in Agra, Uttar Pradesh. Consecutive non-pregnant females of reproductive age consulting for pelvic symptoms especially regarding conception were included in the study. Women who had received antibiotics in the preceding month were excluded. A questionnaire covering demo- graphic data, contraceptive use, recent antibiotic use, sexual history, medical history and history of vaginitis and infertility was completed for each of the participants, and the symptoms that precipitated the patient's visit were recorded. An informed consent was taken from every recruited patient.

A sample obtained from the outer third of the vaginal wall with a dry cotton wool-tipped swab was tested for $\mathrm{pH}$. Additional specimens of vaginal discharge were obtained from the posterior fornix for amine test, gram stain, wet mount, $\mathrm{KOH}$ test and cultures for yeasts and bacteria. Cervical specimens were obtained for Neisseria gonorrhoeae.

Laboratory procedures

The $\mathrm{pH}$ was determined using a $\mathrm{pH}$ indicator paper (Macherey- Nagel, Germany) with a pH range of 3.8 \pm 5.8 . For the amine test ("whiff" test) a drop of potassium hydroxide $10 \%$ was added to a sample of vaginal discharge. The test was considered positive if the typical fishy odor was produced. A gram-stained smear was examined by microscopy for the composition of bacterial morpho- types and presence of yeasts and leukocytes. For the diagnosis of bacterial vaginosis the Nugent scoring system was used. ${ }^{[5]}$ This method is based on the quantity of three different bacterial morphotypes: large gram-positive rods, which represent lactoba- cilli; small gram-variable rods, which represent Gardnerella and Bacteroides/Prevotella species; and curved rods, which represent Mobiluncus species. On the basis of these results, the specimen is assigned a score from 0 to 10 , with $1 \pm 3$ as normal, $4 \pm 6$ as intermediate, and $7 \pm 10$ as bacterial vaginosis. Specimens for yeast culture were transported to the laboratory in modified Stuart's medium and plated on Sabouraud dextrose agar; cervical specimens were transported to the laboratory in charcoal transport medium within a few hours and were plated on chocolate agar for the detection of Neisseria gonorrhoeae.

Yeast or Trichomonal vaginitis was identified by means of $10 \%$ potassium hydroxide $(\mathrm{KOH})$ and saline wet mount examinations of vaginal secretions. The presence of pseudohyphae or budding yeast cells was considered diagnostic of candidal infection. The presence of trichomonads with characteristic motility was considered diagnostic of trichomonal infection.

For the presence of aerobic bacterial infections, the vaginal swab was rolled out onto a 5\% sheep blood agar plate and mac concky agar and the plates were incubated at $37^{\circ} \mathrm{C}$ in a $\mathrm{CO} 2$ thermostater. The colonies were read out as per standard protocol.

\section{Results}

A total of 920 patients were enrolled in the study after following inclusion and exclusion criteria. The median age of these women was 29 years (range 23-37). All women were married. No microorganisms were observed in $44.2 \%$ (407/920) of the individuals studied and (513/920) $55.8 \%$ had positive microbiology. One hundred eighty nine $(20.5 \%)$ were positive for bacterial vaginosis which showed highest prevalence among pathogens. Bacterial species like Enterobacteriaceae (13\%), Enterococcus sp (3.5\%), Streptococcus sp. (1.8\%) were also isolated in high percentage in these patients implicating their importance [Table 1]. These bacterial species were also resistant to many routinely used antibiotic drugs. It was also noted during the study that $115 / 513$ (22.4\%) showed two or more pathogenic microorganisms [Table 2].

When we analyzed the statistical association among presence of lactobacilli and polymorphonuclear cells with microorganisms isolated in vaginal swab, we found that absence of lactobacilli or fewer lactobacilli $(+)$ is significantly (p value $\leq 0.05$ ) associated with isolation of bacterial vaginosis, Enterobacteriaceae, Enterococcus sp. and Streptococcus species. Moreover the results also showed significant association with isolation of no microorganism and presence of lactobacilli $(++/+++)$. Presence of polymorphonuclear cells was also associated significantly ( $\mathrm{p}$ value $\leq 0.05$ ) with prevalence of Enterobacteriaceae, Enterococcus sp. and Streptococcus species. There was a significant association between absence of polymorphonuclear cells and presence of any microorganism [Table 3]. 


\section{Tables}

Table 1: Prevalence of pathogenic microorganisms found in lower genital tract in females with pelvic symptoms

\begin{tabular}{|l|l|l|}
\hline Microorganisms isolated & Positive no. $(\mathrm{n})$ & Percent isolation (\%) \\
\hline Bacterial vaginosis & 189 & 20.5 \\
\hline Trichomonas vaginalis & 100 & 10.9 \\
\hline Candida sp. & 55 & 5.9 \\
\hline Enterobacteriaceae & 120 & 13 \\
\hline Enterococcus sp. & 32 & 3.5 \\
\hline Streptococcus sp. & 17 & 1.8 \\
\hline No organism & 407 & 44.2 \\
\hline
\end{tabular}

Table 2: Prevalence of various combinations of mixed infections among symptomatic females

\begin{tabular}{|l|l|l|}
\hline Types of mixed infections & Positive no. (n) & Percent isolation (\%) \\
\hline BV + Trichomonas (T) & 29 & 25.2 \\
\hline BV + Candida (C) & 15 & 13 \\
\hline BV + Aerobic Bacterial infection (ABI) & 13 & 11.3 \\
\hline BV + T + C & 1 & 0.9 \\
\hline BV + T + ABI & 5 & 4.3 \\
\hline BV + C + ABI & 7 & 6.1 \\
\hline T + C & 0 & 0.0 \\
\hline T + ABI & 19 & 16.5 \\
\hline C + ABI & 26 & 22.6 \\
\hline T + C + ABI & 0 & 0.0 \\
\hline Total mixed infections & 115 & \\
\hline
\end{tabular}

Table 3: Statistical association among presence of lactobacilli and polymorphonuclear cells with microorganisms isolated in vaginal swab

\begin{tabular}{|c|c|c|c|c|c|c|}
\hline \multirow[t]{2}{*}{ Microorganism isolated } & \multicolumn{3}{|c|}{ Presence of Lactobacilli } & \multicolumn{3}{|c|}{ Presence of polymorphonuclear cell } \\
\hline & $\begin{array}{l}++/+++ \\
(\mathrm{n}=380)\end{array}$ & $\begin{array}{l}0 /+ \\
(n=540)\end{array}$ & $\mathrm{P}$ value & $\begin{array}{l}++/+++ \\
(n=476)\end{array}$ & $\begin{array}{l}0 /+ \\
(n=444)\end{array}$ & $\mathrm{P}$ value \\
\hline Bacterial vaginosis & $9(2.4 \%)$ & $180(33.3 \%)$ & $<0.05$ & $140(29.4 \%)$ & $49(11.0 \%)$ & \\
\hline Candida sp. & $20(5.3 \%)$ & $35(6.5 \%)$ & & $32(6.7 \%)$ & $23(5.2 \%)$ & \\
\hline Enterobacteriaceae & $7(1.8 \%)$ & $113(21 \%)$ & $<0.05$ & $109(22.9 \%)$ & $11(2.5 \%)$ & $<0.05$ \\
\hline Enterococcus sp. & $2(0.5 \%)$ & $30(5.6 \%)$ & $<0.05$ & $28(5.9 \%)$ & $4(0.9 \%)$ & $<0.05$ \\
\hline No organism & $302(79.5 \%)$ & $105(19.4 \%)$ & & $95(19.9 \%)$ & $312(70.3 \%)$ & \\
\hline
\end{tabular}

\section{Discussion}

The presence of genital co-infections, whether sexually transmitted or not, may have importance in the outcome of many gynecological diseases including infertility. These infections are generally considered the leading preventable cause of infertility worldwide, especially in developing countries. ${ }^{[8]}$ However most of the women generally neglect the disease due to lack of education as well as having misbelieve regarding the improvement of disease without treatment. Our data demonstrate a high rate of preventable infections in these women. Presence of bacterial infections like Enterobacteriaceae, Enterococci and Streptococcus sp. in our study population demonstrate a causative role of these in vaginosis. Many researcher tried to see this association, some author believe that Streptococcus agalactiae should be considered a causative agent for vaginosis ${ }^{[9]}$ However other says that it is mere colonization of genital tract. ${ }^{[10]}$ Casari $\mathrm{E}$ et al also concluded that it is important to culture the vaginal discharge for Streptococcus agalactiae, Enterobacteriaceae and Enterococci. He has also indicated that the prevalence of some microorganisms (Gardenella vaginalis, Enterobacteriaceae and Enterococci, Streptococcus agalactiae) in the population of asymptomatic women with infertility problems need to be better analyzed. Our study also showed maximum cases of Gardenella vaginalis in study population. We found a high rate of mixed infection giving the idea that all women must be extensively searched for these infections.

In the present study, absence of lactobacilli was correlated well with presence of bacterial vaginosis, Enterobacteriaceae, Enterococcus sp. and Streptococcus species. Many authors have seen the association of lack of lactobacilli with presence of Streptococcus agalactiae, E.coli and bacterial vaginosis. ${ }^{[1,11]}$ They also reported the positive association of presence of polymorphs and various pathogenic microorganisms. It was in concordance with our study in which significant association was found with presence of Enterobactiriaceae, Enterococci and Streptococcus. In fact our study demonstrate that lack of lactobacilli and presence of polymorphs in vaginal discharge may be considered as screening parameters for presence of genital infection. 


\section{Conclusion}

Our findings suggest that symptomatic female should be screened for these infections as the consequences may be up to infertility. However the present study is a laboratory based retrospective study, therefore a detailed clinic-microbiological study is required to demonstrate the association of these infections with infertility.

\section{Acknowledgement} support.

The authors thank NIMS University, Jaipur for their guidance and Dr Deepa Dhawan for their technical

\section{References}

[1] Casari E, Ferrario A, Morenghi E, Montanelli A. Gardnerella, Trichomonas vaginalis, Candida, Chlamydia trachomatis, Mycoplasma hominis and Ureaplasma urealyticum in the genital discharge of symptomatic fertile and asymptomatic infertile women. New Microbiologica 2010;33:69-76.

[2] Richard LS. Treatment of Acute Pelvic Inflammatory Disease. Infectious Diseases in Obstetrics and Gynecology 2011:1-13.

[3] Atashili J, Poole C, Ndumbe PM, Adimora AA, Smith JS. Bacterial vaginosis and HIV acquisition: a meta-analysis of published studies. AIDS 2008;22(12):1493-1501.

[4] Amsel R, Totten PA, Spiegel CA, Chen KC, Eschenbach D, Holmes KK. Nonspecific vaginitis. Diagnostic criteria and microbial and epidemiologic associations. Am J Med 1983;74(1):14-22.

[5] Nugent RP, Krohn MA, Hillier SL. Reliability of diagnosing bacterial vaginosis is improved by a standardized method of gram stain interpretation. J Clin Microbiol 1991;29(2):297-301.

[6] McClelland RS. Trichomonas vaginalis Infection: Can We Afford to Do Nothing? The Journal of Infectious Diseases 2008;197:487-9.

[7] Wilkinson D, Ndovela N, Harrison A, Lurie M, Connolly C, A Sturm W. Family planning services in developing countries: an opportunity to treat asymptomatic and unrecognised genital tract infections? Genitourin Med 1997;73:558-560.

[8] Sciarra J. Infertility: an international health problem. Int J Gynaecol Obstet 1994;46:155-63.

[9] Honig E, Mouton JW, Van Der Meijden WI. The epidemiology of vaginal colonisation with group B streptococci in a sexually transmitted disease clinic. Eur. J. Obste. Gynecol. Reprod. Biol. 2002;105(2):177- 180.

[10] Rowen D. Streptococci and the genital tract. Int. J. STD. AIDS 1993;4:63-66.

[11] Hillier SL. Diagnostic microbiology of bacterial vaginosis. Am. J. Obstet. Gynecol 1993;169:455- 459. 